\title{
Unilateral Neurogenic Pulmonary Edema Following Cerebral Aneurysmal Clipping: An Atypical Presentation of Hypoxemia
}

\author{
Deepti Srinivas ${ }^{1} \quad K^{\prime}$ eshavan Venkatesh ${ }^{2} \quad$ Archisha Kapoor $^{1} \quad$ Rashmi Patil $^{1}$
}

\author{
${ }^{1}$ Department of Neuroanaesthesia and Critical care, Apollo \\ Hospitals, Bangalore, India \\ 2Department of Anaesthesiology and Critical Care, Khoula Hospital, \\ Muscat, Oman
}

J Neuroanaesthesiol Crit Care 2022;9:66-67.

Unilateral pulmonary edema secondary to neurogenic cause is a rare entity. ${ }^{1}$ We report such a case following cerebral aneurysmal clipping. A 47-year-old female with no comorbidities presented with headache of 6 days' duration. Computed tomography (CT) brain showed right Sylvian fissure bleed and subarachnoid hemorrhage (SAH) (Fischer grade 2). Cerebral angiogram revealed a right middle cerebral artery (MCA) aneurysm with moderate vasospasm in proximal M2 segment. Clinically, she was World Federation of Neurological Surgeons (WFNS) grade I.

Chest X-ray (CXR) was unremarkable, and echocardiogram showed hypokinetic basal septum, and basal and midinferior segments with a left ventricular ejection fraction of 50\%. Right pterional craniotomy and clipping of the aneurysm was accomplished uneventfully under general anesthesia. The patient was extubated at the end of surgery. Cerebral angiogram repeated on postoperative day (POD) one showed moderate-to-severe vasospasm in the right proximal M2 segment and was managed with intra-arterial milrinone $8 \mathrm{mg}$ over 30 minutes in right internal carotid artery (ICA). This was followed by intravenous (IV) nimodipine infusion $1 \mathrm{mg} / \mathrm{h}$, increased to $2 \mathrm{mg} / \mathrm{h}$ for a period of 72 hours. Mean arterial pressure was maintained at 90 to $100 \mathrm{~mm} \mathrm{Hg}$ with titrated doses of phenylephrine infusion.

On POD six, she became drowsy and tachypneic (respiratory rate of $45-50$ breaths per minute). She was afebrile and had no neurological deficits on examination. Auscultation revealed fine crepitations in bilateral lung fields, more on the right side. Diagnosis of pulmonary edema was made. Lung ultrasound revealed extensive B-lines on the right side, and CXR demonstrated heterogenous dense opacities only in the right lung with prominent vascular markings ( - Fig. 1A),

published online

November 19, 2021
DOI https://doi.org/

$10.1055 / \mathrm{s}-0041-1739346$ ISSN 2348-0548
Address for correspondence Deepti Srinivas, PDF, Department of Neuroanaesthesia and Critical care, Apollo Hospitals, BG Road, Bangalore 560076, Karnataka, India (e-mail: bsdeepti@gmail.com).

suggestive of pulmonary edema. She was initially managed on noninvasive ventilation. However, due to progressive dyspnea and hypoxemia $\left(\mathrm{pO}_{2}=60 \mathrm{~mm} \mathrm{Hg}\right.$ on $\left.\mathrm{FiO}_{2} 0.5\right)$, she was intubated and ventilated on pressure control mode with positive end expiratory pressure (PEEP) of $10 \mathrm{~cm}$ of $\mathrm{H}_{2} \mathrm{O}$. Her fluid balance was positive by $1500 \mathrm{~mL}$ over 3 days (POD 4-6). Inferior vena cava (IVC) diameter was $17 \mathrm{~mm}$ and less than $50 \%$ collapsing, suggestive of optimum intravascular volume status. Echocardiogram was comparable to preoperative status. Her plasma protein levels were normal. She was started on IV furosemide $10 \mathrm{mg}$ thrice a day. Her tracheal aspirate was negative for bacterial infection. The total leukocyte count (TLC) was $18 \times 10^{9} / \mathrm{L}$, increased from a preoperative baseline of $13 \times 10^{9} / \mathrm{L}$. CT brain showed subacute infarct in the right temporal lobe, corresponding to the MCA territory.

The patient showed symptomatic improvement within 6 hours. Radiologically, the opacities started clearing within 24 hours and consistently improved over the next 4 days $(-$ Fig. 1B, C). She was weaned from the ventilator and extubated 3 days postintubation and discharged from the ICU on POD 12.

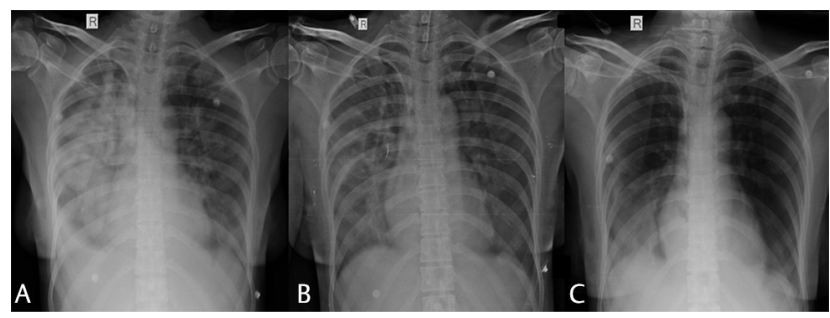

Fig. 1 (A-C) Chest X-ray on postoperative days 6, 8, and 10, respectively, showing heterogenous opacities initially and gradual clearing within 4 days. (c) 2021. Indian Society of Neuroanaesthesiology and Critical Care. All rights reserved.

This is an open access article published by Thieme under the terms of the Creative Commons Attribution-NonDerivative-NonCommercial-License, permitting copying and reproduction so long as the original work is given appropriate credit. Contents may not be used for commercial purposes, or adapted, remixed, transformed or built upon. (https://creativecommons.org/licenses/by-nc-nd/4.0/).

Thieme Medical and Scientific Publishers Pvt. Ltd. A-12, 2nd Floor, Sector 2, Noida-201301 UP, India 
The reported incidence of neurogenic pulmonary edema (NPE) associated with aneurysmal SAH is around $8 \%$. The onset can be as late as up to a week. When associated with neurological disorders, the pulmonary infiltrates are predominantly bilateral. ${ }^{2,3}$ The pathophysiology of NPE is contentious with various proposed theories, ${ }^{4}$ most of which are derived from catecholamine surge due to the central nervous system (CNS) insult, leading to changes in cardiopulmonary hemodynamics and Starling forces. Other factors such capillary leak and myocardial dysfunction can also play a role.

To our knowledge, there is only one report of unilateral NPE occurrence following cerebral aneurysmal clipping to date. ${ }^{1}$ The differential diagnoses considered included aspiration pneumonia, cardiogenic pulmonary edema, fluid overload-induced pulmonary edema, and hypoalbuminemia. The thin and watery quality of tracheal secretions and absence of microorganisms on culture made the diagnosis of infectious origin unlikely. The speedy recovery observed clinically and radiologically with mechanical ventilation, high PEEP and diuretics make pulmonary edema highly plausible. The calculated fluid overload percentage $\mathrm{e}^{5}$ was $<2 \%$, which is negligible. Normal IVC diameter also precludes fluid overload as the primary etiology. The $2 \mathrm{D}$ echocardiogram changes were consistent with SAH-associated changes. Moreover, cardiogenic and fluid overload-induced pulmonary edema is always bilateral. The temporal association of development of vasospasm as seen on angiogram, and development of sequelae in the form of pulmonary edema further establishes its neurogenic nature.

NPE is a diagnosis of exclusion, which responds to prompt treatment without any sequelae. Our case was a rare occurrence of unilateral presentation, which added ambiguity in diagnosis. Hence, when associated with neurological pathology, a high index of suspicion with timely diagnosis and management will ensure resolution and good outcome.

\section{Conflict of Interest}

None declared.

\section{References}

1 Durga P, Jonnavithula N, Panigrahi MK, Mantha S. Unilateral neurogenic pulmonary oedema: An unusual cause for post-operative respiratory dysfunction following clipping of ruptured intracranial aneurysm. Indian J Anaesth 2012;56(1): 58-61

2 Lin CN, Howng SL, Kuo TH, Hwang SL, Kao EL. [Neurogenic pulmonary edema: five cases report]. Gaoxiong Yi Xue Ke Xue Za Zhi 1992;8(9):510-519

3 Tsai YS, Wang SJ, Shih HC, et al. Unilateral pulmonary edema during general anesthesia-report of two cases. Acta Anaesthesiol Sin 1997;35(3):175-180

4 Davison DL, Terek M, Chawla LS. Neurogenic pulmonary edema. Crit Care 2012;16(2):212

5 Claure-Del Granado R, Mehta RL. Fluid overload in the ICU: evaluation and management. BMC Nephrol 2016;17(1):109 